\title{
ORIGINAL ARTICLE \\ Intervention with a caspase-1 inhibitor reduces obesity-associated hyperinsulinemia, non-alcoholic steatohepatitis and hepatic fibrosis in LDLR-/-.Leiden mice
}

\author{
MC Morrison ${ }^{1,2,3}$, P Mulder $^{1}$, K Salic ${ }^{1}$, J Verheij ${ }^{4}$, W Liang ${ }^{1}$, W van Duyvenvoorde ${ }^{1}$, A Menke ${ }^{5}$, T Kooistra ${ }^{1}$, R Kleemann ${ }^{1}$ and PY Wielinga ${ }^{1}$
}

\begin{abstract}
BACKGROUND/OBJECTIVES: Non-alcoholic steatohepatitis (NASH) is a serious liver condition, closely associated with obesity and insulin resistance. Recent studies have suggested an important role for inflammasome/caspase-1 in the development of NASH, but the potential therapeutic value of caspase- 1 inhibition remains unclear. Therefore, we aimed to investigate the effects of caspase- 1 inhibition in the ongoing disease process, to mimic the clinical setting.

SUBJECTS/METHODS: To investigate effects of caspase-1 inhibition under therapeutic conditions, male LDLR-/-.Leiden mice were fed a high-fat diet (HFD) for 9 weeks to induce a pre-diabetic state before start of treatment. Mice were then continued on HFD for another 12 weeks, without (HFD) or with (HFD-YVAD) treatment with the caspase-1 inhibitor Ac-YVAD-cmk (40 mg kg ${ }^{-1}$ per day).

RESULTS: Nine weeks of HFD feeding resulted in an obese phenotype, with obesity-associated hypertriglyceridemia, hypercholesterolemia, hyperglycemia and hyperinsulinemia. Treatment with Ac-YVAD-cmk did not affect further body weight gain or dyslipidemia, but did attenuate further progression of insulin resistance. Histopathological analysis of livers clearly demonstrated prevention of NASH development in HFD-YVAD mice: livers were less steatotic and neutrophil infiltration was strongly reduced. In addition, caspase- 1 inhibition had a profound effect on hepatic fibrosis, as assessed by histological quantification of collagen staining and gene expression analysis of fibrosis-associated genes Col1a1, Acta2 and Tnfa.

CONCLUSIONS: Intervention with a caspase-1 inhibitor attenuated the development of NASH, liver fibrosis and insulin resistance. Our data support the importance of inflammasome/caspase-1 in the development of NASH and demonstrate that therapeutic intervention in the already ongoing disease process is feasible.
\end{abstract}

International Journal of Obesity (2016) 40, 1416-1423; doi:10.1038/ijo.2016.74

\section{INTRODUCTION}

Non-alcoholic fatty liver disease (NAFLD) has become the most common cause of chronic liver disease in Western countries, and its prevalence continues to rise in parallel with increasing rates of obesity and type 2 diabetes, to which it is strongly related. ${ }^{1,2}$ NAFLD encompasses a spectrum of liver disease that ranges from the clinically benign intrahepatic accumulation of lipids (steatosis) to the more progressive non-alcoholic steatohepatitis (NASH), which is characterized by the presence of hepatic inflammation, ballooning and fibrosis. ${ }^{3}$ NASH is a serious liver disease that can further progress to cirrhosis and hepatocellular carcinoma, ${ }^{4}$ and is projected to become the leading indication for liver transplantation in the next several years, ${ }^{5}$ particularly since there is currently no approved pharmacological therapy for NAFLD/NASH.

A crucial factor in the pathogenesis of NAFLD is considered to be the chronic low-grade inflammatory state that characterizes metabolic overload and obesity. ${ }^{6}$ It is assumed that this metabolically-induced inflammation results from excess nutrient and energy intake and originates in the adipose tissue. ${ }^{7}$ A key component in metabolic inflammation is the NLRP3 inflammasome, a multi-protein danger-sensing complex that is thought to form the crossroads between metabolism and inflammation. ${ }^{8}$ This large cytoplasmic complex comprises the receptor protein NLRP3, which can be activated by classical pro-inflammatory signals as well as by metabolic signals such as free fatty acids., ${ }^{9,10}$ Activation of NLRP3 facilitates the recruitment and activation of caspase-1, which cleaves the biologically inactive precursors of the cytokines IL-1 $\beta$ and IL-18 into their mature, pro-inflammatory counterparts. ${ }^{11}$

Several studies provide indication that inflammasome activation indeed plays an important role in the development of NAFLD. Analyses of human liver biopsies have shown that mRNA expression of NLRP3 inflammasome-related proteins (for example, NLRP3, caspase-1, pro-IL-1 $\beta$, pro-IL-18) is increased in livers of NASH patients ${ }^{9,12}$ and that their expression correlates with the extent of fibrosis development. ${ }^{12}$ In line with this, studies in experimental models of NASH have shown that caspase- 1 is activated in NASH livers ${ }^{9,13,14}$ and that development of NASH is clearly reduced in mice in which inflammasome-associated genes are knocked out, ${ }^{12-14}$ whereas constitutive hyperactivation of the NLRP3 inflammasome results in severe liver inflammation and fibrosis. ${ }^{15}$

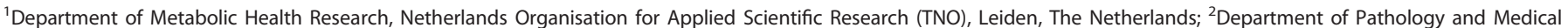

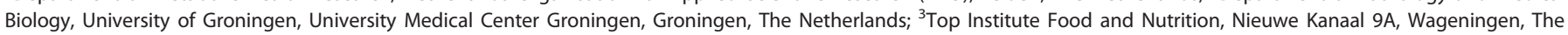

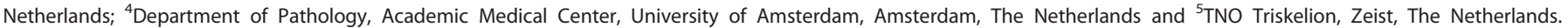

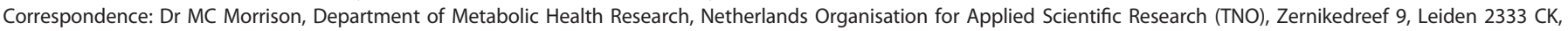
The Netherlands. 
However, since most of these studies were performed in genetically engineered mouse strains that are completely deficient in inflammasome-associated genes like NLRP3 or caspase-1, the potential therapeutic value of inflammasome inhibition in the ongoing disease process, that is, conditions reflective of the clinical setting, remains unclear. Therefore, we aimed to investigate the effect of treatment with an inflammasome inhibitor on the development of obesity-associated NAFLD. To this end, we used LDLR-/-.Leiden mice, a translational model for obesity-associated diseases. ${ }^{16}$ When fed a high-fat diet (HFD), these mice develop diet-induced obesity, metabolic inflammation and insulin resistance that progresses to development of type 2 diabetes and NASH. ${ }^{17,18}$ We treated HFD-fed LDLR-/-.Leiden mice with the caspase-1 inhibitor Ac-YVAD-cmk, ${ }^{19-21}$ starting treatment once early disease symptoms were present, to allow us to study the effects of inflammasome inhibition in a therapeutic setting.

\section{MATERIALS AND METHODS}

\section{Animals}

Animal experiments were approved by an independent Committee on the Ethics of Animal Experiments (DEC-Zeist, The Netherlands; Permit Number: 3216). Male LDLR-/-.Leiden mice were kept on chow (Sniff-R/M-V1530, Uden, the Netherlands) until the start of the study (at 12-14 weeks of age). The mice were then matched into three groups ( $n=15 /$ group) based on body weight (sample size based on previously performed pilot studies). The first group received a low-fat reference diet (LFD, $10 \mathrm{kcal} \%$ fat from lard; D12450B, Research Diets, New Brunswick, NJ, USA). The other two groups were fed a high-fat diet (HFD, $45 \mathrm{kcal} \%$ fat from lard; D12451, Research Diets) for 9 weeks, after which the intervention was started. Both groups continued on HFD for the remainder of the study, and from $t=9$ weeks onwards group 3 (HFD-YVAD) received daily intraperitoneal injections with the caspase-1 inhibitor Ac-YVAD-cmk $\left(40 \mathrm{mg} \mathrm{kg}^{-1}\right.$; Bachem, Weil a.Rhein, Germany). Earlier (pilot) studies showed that vehicle (phosphate-buffered saline) intraperitoneal injections did not affect body weight, organ integrity or plasma biochemistry relative to non-injected controls. For ethical reasons, the current study, therefore, did not include a control group receiving vehicle injections, in line with the Three Rs policy and in agreement with the ethical committee that assesses all study protocols. Body weight (individual) and food intake (per cage) were measured every 3 weeks. Body fat percentage was determined by EchoMRI (EchoMRI-LLC, Houston, TX, USA). Tail blood samples (for EDTA plasma) were taken at regular intervals after a 5 -h fast. At $t=20$ weeks, an intraperitoneal glucose tolerance test (ipGTT) was performed ( $n=8 /$ group, randomly selected). For this, mice were fasted for $5 \mathrm{~h}$ before they were injected with $33 \mathrm{mg}$ glucose (comparable to $1 \mathrm{~g}$ per $\mathrm{kg}$ body weight based on average body weight of $33 \mathrm{~g}$ in the LFD control group). Tail blood samples were taken before $(t=0)$ and at $5,15,30,60$ and 120 min post injection to determine blood glucose and plasma insulin levels. At $t=21$ weeks, the mice were killed by $\mathrm{CO}_{2}$, terminal blood (for serum collection) was obtained via cardiac puncture and isolated organs were weighed.

\section{Blood and plasma analyses}

Blood glucose was measured immediately using a hand-held glucose analyzer (Freestyle Disectronic, Vianen, the Netherlands). Plasma insulin levels were determined by ELISA (Mercodia, Uppsala, Sweden). Total plasma cholesterol and triglyceride levels were measured with enzymatic assays (Roche Diagnostics, Almere, the Netherlands). Plasma alanine aminotransferase levels were measured using a spectrophotometric activity assay (Reflotron-Plus, Roche).

\section{Histological analysis of adipose tissue and liver}

White adipose tissue (WAT) inflammation was assessed in the three major WAT depots (inguinal, mesenteric and epididymal). Tissues were formalinfixed and paraffin-embedded, and cross-sections $(5 \mu \mathrm{m})$ were stained with hematoxylin-phloxine-saffron. WAT inflammation was quantified blindly by counting the number of crown-like structures (CLSs) in 3 non-overlapping fields (at x100 magnification, view size $3.1 \mathrm{~mm}^{2}$ ) for each depot, expressed as number of CLSs per $\mathrm{mm}^{2}$.
Formalin-fixed and paraffin-embedded cross-sections $(5 \mu \mathrm{m})$ of the median lobe were stained with hematoxylin and eosin and scored blindly by a board-certified pathologist using an adapted grading method for human NASH. ${ }^{16}$ Briefly, two cross-sections/mouse were examined and the level of microvesicular and macrovesicular steatosis was determined relative to the liver area analyzed (expressed as a percentage). Hepatic inflammation was assessed by counting the number of inflammatory foci per field at a $\times 100$ magnification (view size $3.1 \mathrm{~mm}^{2}$ ) in five nonoverlapping fields per specimen, expressed as the average number of foci per field. Immunohistochemical staining for myeloperoxidase (MPO; ab9535; Abcam, Cambridge, UK) was used to identify neutrophils, and the number of MPO-positive inflammatory clusters was counted as described above. Fibrosis was assessed histochemically by Picro-Sirius Red staining (Chroma, WALDECK-Gmbh, Münster, Germany). Collagen content was quantified using ImageJ software (version 1.48, NIH, Bethesda, MD, USA) to assess the area of liver tissue that was positively stained (expressed as the percentage of total tissue area). In addition, development of fibrosis was assessed by a pathologist to gain insight into the distribution pattern of the collagen and to quantify the percentage of pericellular fibrosis specifically (expressed as the percentage of pericellular fibrosis relative to the total perisinusoidal area).

\section{Analysis of liver lipids}

Liver lipids were analyzed by high-performance thin-layer chromatography as described previously. ${ }^{22}$ In brief, lipids were extracted from liver homogenates using methanol and chloroform following the Bligh and Dyer method, ${ }^{23}$ after which they were separated by high-performance thin-layer chromatography on silica gel plates. Lipid spots were stained with color reagent $\left(5 \mathrm{~g}\right.$ of $\mathrm{MnCl}_{2} 4 \mathrm{H}_{2} \mathrm{O}, 32 \mathrm{ml}$ of $95-97 \% \mathrm{H}_{2} \mathrm{SO}_{4}$ added to $960 \mathrm{ml}$ of $\left.\mathrm{CH}_{3} \mathrm{OH} / \mathrm{H}_{2} \mathrm{O}=1: 1(\mathrm{v} / \mathrm{v})\right)$ and triglycerides, cholesteryl esters and free cholesterol were quantified using TINA version 2.09 software (Raytest, Straubenhardt, Germany). Liver lipids were expressed per mg liver protein, which was determined in the same liver homogenates used for the liver lipid analysis, using the Lowry protein assay. ${ }^{24}$

\section{Hepatic gene expression analyses}

RNA-Bee Total-RNA Isolation Kit (Bio-Connect, Huissen, the Netherlands) was used for RNA extraction. RNA concentration was determined spectrophotometrically using Nanodrop 1000 (Isogen Life Science, De Meern, the Netherlands) and RNA quality was assessed using 2100 Bioanalyzer (Agilent Technologies, Amstelveen, the Netherlands). cDNA was synthesized from $1 \mu \mathrm{g}$ of RNA using a High-Capacity RNA-to-cDNA Kit (Life Technologies, Bleiswijk, the Netherlands). Transcripts were quantified using TaqMan Gene Expression Assays (Life Technologies) and the following primer/probe-sets: Emr1 (Mm00802529_m1), Col1a1 (Mm00801666_g1), Tnfa (Mm00443258_m1), Acta2 (Mm01546133_m1) and the endogenous control Ppif (Mm01273726_m1). Changes in gene expression were calculated using the comparative $C t(\Delta \Delta C t)$ method and expressed as fold-change relative to LFD as described previously. ${ }^{25}$

\section{Statistical analysis}

Data were analyzed with SPSS 22.0 (IBM, Armonk, NY, USA). Differences between groups were analyzed by one-way analysis of variance followed by LSD post-hoc analysis. Variables with unequal variances were analyzed by analysis of variance (Brown-Forsythe) and Dunnett's T3 post-hoc test. Non-normally distributed variables were analyzed by non-parametric Kruskal-Wallis followed by Mann-Whitney $U$-test. To test the hypothesis that treatment with Ac-YVAD-cmk may attenuate development of WAT inflammation, insulin resistance and NASH, a one-sided $P<0.05$ was considered statistically significant. All results are shown as mean \pm s.e.m.

\section{RESULTS}

To investigate the effects of caspase- 1 inhibition under therapeutic conditions, LDLR-/-.Leiden mice were fed a HFD for 9 weeks to induce a pre-diabetic state before the start of treatment with the caspase- 1 inhibitor Ac-YVAD-cmk. This HFD feeding resulted in an obese phenotype with obesity-associated hypertriglyceridemia, hypercholesterolemia, hyperglycemia and hyperinsulinemia (Table 1, all $P<0.05$ compared with $t=0$ ). As a 
reference, a LFD-fed group of LDLR-/-.Leiden mice was included that developed a milder obese pre-diabetic phenotype (Table 1).

Caspase-1 inhibition does not affect obesity or dyslipidemia, but does improve adipose tissue inflammation and insulin sensitivity. After these first 9 weeks of HFD feeding, treatment with Ac-YVADcmk was started in half of the HFD-fed animals (HFD-YVAD), whereas the other 15 mice remained on the HFD alone (HFD). The LFD reference group continued on the LFD until the end of the study. Ac-YVAD-cmk treatment did not affect food intake (average food intake during treatment period: $12.5 \pm 0.3 \mathrm{kcal}$ per mouse per day in HFD, $11.9 \pm 0.8 \mathrm{kcal}$ per mouse per day in HFD-YVAD, not significant), HFD-induced body weight gain (Figure 1a), body fat percentage (Figure 1b) or dyslipidemia (Supplementary Figure 1). Refined histological analysis of WAT quality revealed that HFD feeding resulted in WAT inflammation in the epididymal depot, as reflected by a clear presence of CLS in this depot specifically (Figure 1c). Ac-YVAD-cmk treatment

Table 1. Pre-treatment characteristics

\begin{tabular}{|c|c|c|c|}
\hline & \multirow{2}{*}{$\begin{array}{l}\mathrm{t}=0 \\
\text { Chow }\end{array}$} & \multicolumn{2}{|c|}{$\mathrm{t}=9$ Weeks } \\
\hline & & $L F D$ & $H F D$ \\
\hline Body weight (g) & $26.3 \pm 0.3^{a}$ & $32.3 \pm 0.9^{b}$ & $39.0 \pm 1.0^{c}$ \\
\hline Plasma cholesterol (mм) & $5.4 \pm 0.1^{a}$ & $16.1 \pm 2.0^{b}$ & $16.8 \pm 1.1^{\mathrm{b}}$ \\
\hline Plasma triglycerides (mm) & $1.0 \pm 0.0^{\mathrm{a}}$ & $2.6 \pm 0.4^{b}$ & $2.6 \pm 0.2^{b}$ \\
\hline Blood glucose (mм) & $6.5 \pm 0.1^{a}$ & $6.5 \pm 0.4^{a}$ & $8.5 \pm 0.2^{b}$ \\
\hline Plasma insulin ( $\mathrm{g} \mathrm{ml}^{-1}$ ) & $1.1 \pm 0.1^{a}$ & $2.2 \pm 0.4^{\mathrm{b}}$ & $5.2 \pm 0.5^{c}$ \\
\hline
\end{tabular}

All data are mean \pm s.e.m. Means in a row with superscripts without common letter differ significantly $(P<0.05)$. reduced $(-41 \%, P<0.05)$ the presence of $C L S$ in epididymal WAT (Figure 1d). This reduction in WAT inflammation was observed in the absence of an effect of Ac-YVAD-cmk on WAT mass or the distribution of WAT mass over the epididymal, mesenteric or inguinal depots (Supplementary Figure 2).

The observed improvement in WAT quality was accompanied by improved insulin sensitivity in HFD-YVAD mice. The HFDinduced increases in fasting glucose (Figure 2a) and fasting insulin levels (Figure 2b) were significantly reduced in HFD-YVAD mice (absolute blood glucose and plasma insulin levels are shown in Supplementary Figure 3). An ipGTT performed at $t=20$ weeks (after 11 weeks of treatment) further confirmed these beneficial effects (Figures $2 c$ and d, showing the delta changes in glucose and insulin post injection). In HFD mice, the glucose injection resulted in a rapid increase in blood glucose, with peak levels reached after $15 \mathrm{~min}(17.8 \pm 1.4 \mathrm{~mm})$, after which they gradually decreased to $12.3 \pm 0.8 \mathrm{~mm}$ at $120 \mathrm{~min}$ post injection. This glucose response was slightly more pronounced than that of LFD mice. In HFD-YVAD, the increase in blood glucose was much slower and the peak was not reached until $60 \mathrm{~min}$ post injection $(15.7 \pm 1.2 \mathrm{~mm})$, after which it decreased to $9.9 \pm 0.8 \mathrm{~mm}$ at $t=120 \mathrm{~min}$. The area under the curve did not differ significantly between HFD and HFD-YVAD animals (not shown). Although LFD mice needed only a slight increase in insulin to clear the glucose, the glucose injection triggered a strong and rapid insulin response in HFD mice, with plasma insulin levels increasing from $14.3 \pm 3.3 \mathrm{ng} \mathrm{ml}^{-1}$ at baseline, to $23.8 \pm 6.7 \mathrm{ng} \mathrm{ml}^{-1}$ at 15 min after the injection. From $t=15$ min onward, plasma insulin levels then gradually declined back to baseline levels $\left(15.1 \pm 5.0 \mathrm{ng} \mathrm{ml}^{-1}\right)$. Remarkably, HFD-YVAD mice showed no insulin response to the injected glucose, with insulin levels remaining comparable to baseline levels for the duration of the GTT. Together, these data indicate improved glucose tolerance and insulin sensitivity in Ac-YVAD-cmk-treated mice.

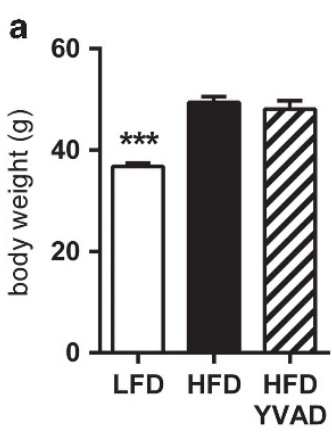

d

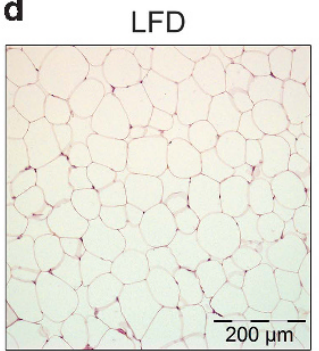

b
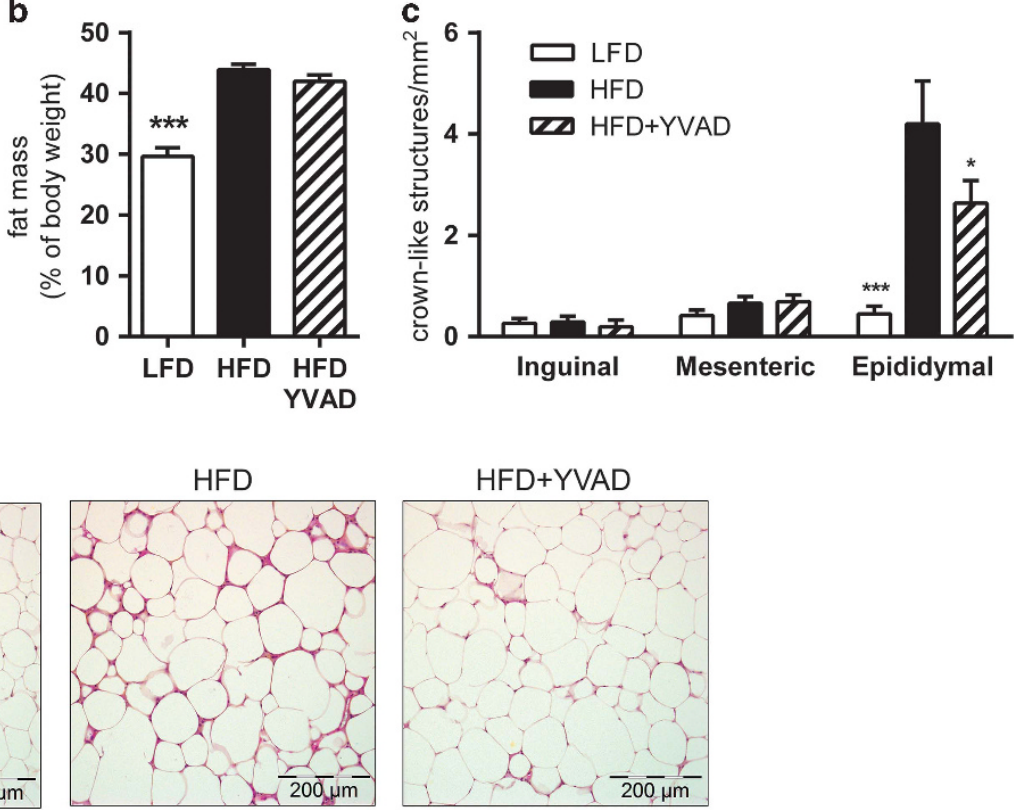

Figure 1. Effects of caspase-1 inhibition on high-fat diet (HFD)-induced body weight, adiposity and adipose tissue inflammation. LDLR-/-. Leiden mice were fed a HFD for 21 weeks $(n=15)$ or HFD+caspase-1 inhibitor Ac-YVAD-cmk $\left(40 \mathrm{mg} \mathrm{kg}^{-1}\right.$ daily) starting treatment after 9 weeks of HFD (HFD+YVAD; $n=15$ ). Low-fat diet (LFD)-fed mice $(n=15)$ were included as a reference. (a) HFD feeding significantly induced body weight relative to LFD, which was not affected by caspase-1 inhibition. (b) Body fat percentage was induced by HFD and was not affected in HFD+YVAD mice. (c) HFD induced pronounced adipose tissue inflammation specifically in the epididymal depot, which tended to be reduced in HFD+YVAD mice. (d) Representative photomicrographs of HPS-stained epididymal adipose tissue sections. All data are from the $t=21$ weeks time point and are mean \pm s.e.m. ${ }^{*} P<0.05,{ }^{* * *} P<0.001$ compared with HFD. 

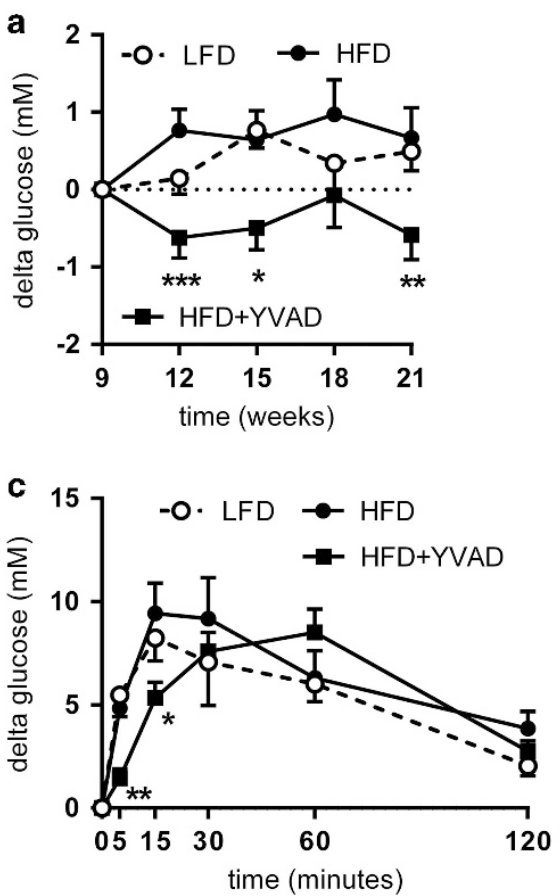
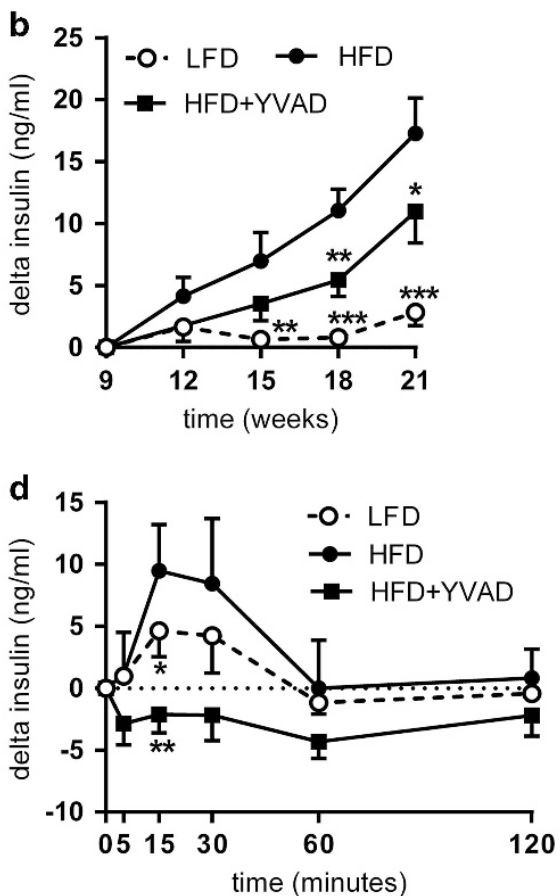

Figure 2. Effects of caspase-1 inhibition on fasting glucose and insulin and glucose tolerance. LDLR-/-.Leiden mice were fed a high-fat diet for 21 weeks (HFD; $n=15)$ or HFD+caspase-1 inhibitor Ac-YVAD-cmk (40 mg kg ${ }^{-1}$ daily) starting treatment after 9 weeks of HFD (HFD+YVAD; $n=15)$. Low-fat diet (LFD)-fed mice $(n=15)$ were included as a reference. (a) HFD-induced increases in fasted blood glucose relative to treatment start ( $t=9$ weeks) were reduced in HFD+YVAD. (b) HFD-induced increases in fasted plasma insulin relative to treatment start ( $t=9$ weeks) were reduced in HFD+YVAD. (c) The glucose response to an intraperitoneal glucose tolerance test (ipGTT; performed at $t=20$ weeks) was modified by caspase-1 inhibition. (d) HFD mice showed a clear insulin response during the ipGTT, whereas HFD+YVAD mice showed no response in plasma insulin. Data are mean \pm s.e.m. ${ }^{*} P<0.05,{ }^{*} P<0.01,{ }^{* *} P<0.001$ compared with HFD at the same time point.

\section{Caspase-1 inhibition improves hepatic steatosis}

To investigate whether these improvements in glucose handling are accompanied by an effect on the development of hepatic steatosis, we analyzed liver cross-sections (Figure 3a) using an adapted scoring system for human NASH. ${ }^{16}$ We observed pronounced hepatic steatosis in HFD-fed animals, with $48.5 \pm 5.6 \%$ of hepatocytes affected by macrovesicular steatosis (Figure $3 \mathrm{~b}$ ) and $40.0 \pm 5.4 \%$ affected by microvesicular steatosis (Figure $3 \mathrm{c}$ ). Although treatment with Ac-YVAD-cmk did not affect development of macrovesicular steatosis ( $54.5 \pm 3.6 \%$, Figure $3 \mathrm{~b}$ ), microvesicular steatosis was significantly reduced $(27.8 \pm 5.6 \%, P<0.01$, Figure $3 c$ ) in livers of HFD-YVAD mice compared with HFD mice. Liver lipid analysis by high-performance thin-layer chromatography revealed that Ac-YVAD-cmk treatment tended to reduce the accumulation of triglycerides in the liver $\left(233.2 \pm 10.0 \mu \mathrm{g} \mathrm{mg}^{-1}\right.$ liver protein in HFD, $205.3 \pm 13.8 \mu \mathrm{g} \mathrm{mg}^{-1}$ liver protein in HFDYVAD, $P=0.08$, Figure $3 \mathrm{~d}$ ), whereas the accumulation of cholesterol (in both free and esterified form) was not affected by the treatment (Supplementary Figure 4).

Caspase-1 inhibition reduces hepatic inflammation

In addition to the observed reduction in hepatic steatosis, Ac-YVAD-cmk had a strong effect on plasma alanine aminotransferase, a marker of hepatocellular damage (HFD: $390.5 \pm 16.4 \mathrm{U} \mathrm{I}^{-1}$, HFD-YVAD: $237.0 \pm 35.7 \mathrm{UI}^{-1}, P<0.05$, Figure 4a). To investigate whether this was reflected by a reduction in NASH development, we next analyzed hepatic inflammation, a defining characteristic of NASH that can be observed histologically as the presence of inflammatory cell foci. Analysis of the number of these inflammatory cell foci showed marked lobular inflammation in HFD mice (3.6 \pm 0.6 foci per $\times 100$ field, Figure 4b), whereas AcYVAD-cmk treatment strongly reduced $(-51 \%)$ the number of these inflammatory cell clusters $(1.8 \pm 0.3$ foci per $x 100$ field, $P<0.01$, Figure $4 \mathrm{~b})$. As hepatic gene expression analysis for the macrophage marker Emr1 (F4/80) indicated that this antiinflammatory effect of Ac-YVAD-cmk was not due to an effect on macrophages (Figure 4c), we next investigated whether AcYVAD-cmk may have an effect on neutrophilic cells, the influx of which is considered a hallmark of human NASH. ${ }^{22,26,27}$ Immunohistochemical staining for the neutrophil marker MPO showed distinct presence of neutrophils in HFD animals, which was clearly reduced in HFD-YVAD mice (Figure 4d), as is also evident from the quantification of the number of MPO-positive inflammatory foci per field (Figure 4e). In line with these results, we observed a profound reduction (43\%) in the mRNA levels of the proinflammatory cytokine Tnfa (TNF- $a)$ in HFD-YVAD mice $(1.0 \pm 0.14$ in HFD, $0.6 \pm 0.06$ in HFD-YVAD, $P<0.05$, Figure $4 f$ ).

\section{Caspase-1 inhibition reduces hepatic fibrosis}

As hepatocellular damage and hepatic inflammation are thought to drive the development of hepatic fibrosis, we next questioned whether Ac-YVAD-cmk treatment may also reduce fibrosis development. Picro-sirius red staining for collagen content revealed the presence of periportal and pericellular fibrosis without bridging in HFD, which was attenuated by Ac-YVADcmk. Automated quantification of the area stained positively for collagen showed a strong reduction in the total fibrotic area $(-65 \%)$ in HFD-YVAD mice compared with HFD $(1.14 \pm 0.28$ in HFD, $0.40 \pm 0.04$ in HFD-YVAD, $P<0.05$, Figure $5 b$ ). The pathologistassessed percentage of pericellular fibrosis specifically was also lower in HFD-YVAD, although this effect was not statistically significant $(1.4 \pm 0.57$ in LFD, $2.3 \pm 0.73$ in HFD, $1.3 \pm 0.51$ in HFDYVAD, $P=0.14$ for HFD-YVAD compared with HFD). Hepatic gene expression analysis of Col1a1 confirmed the observed effect on 
a
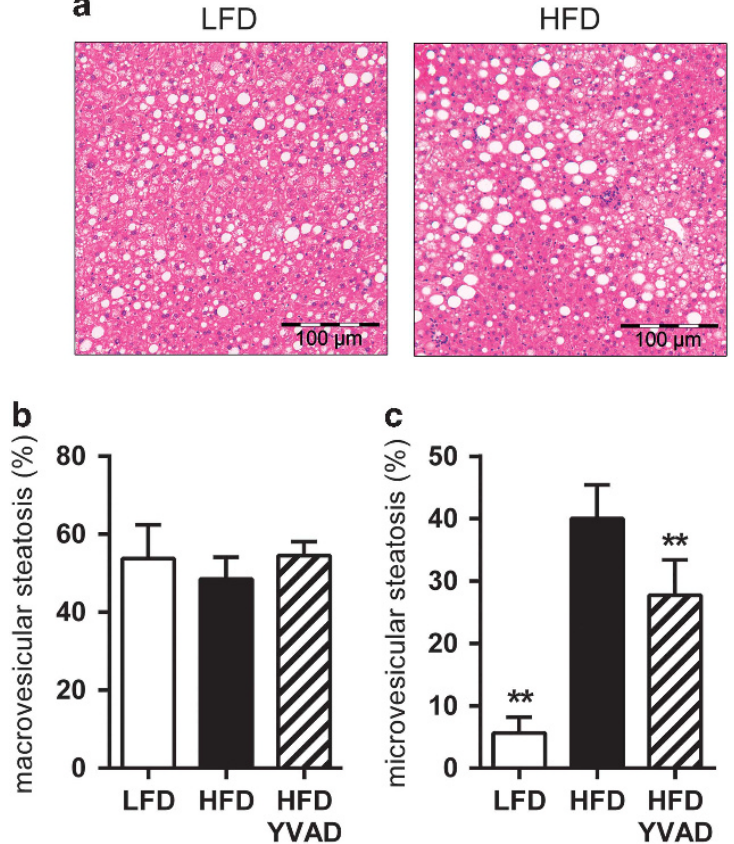

c

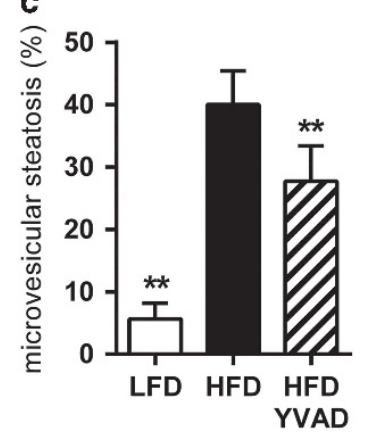

HFD+YVAD

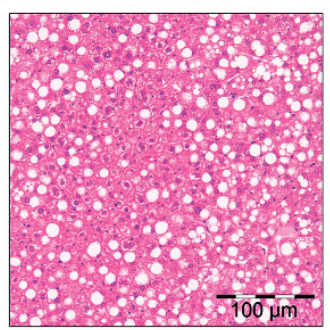

d

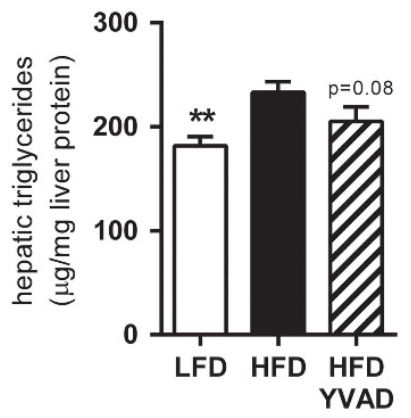

Figure 3. Effects of caspase-1 inhibition on hepatic steatosis. LDLR-/-.Leiden mice were fed a high-fat diet for 21 weeks (HFD; $n=15$ ) or HFD +caspase-1 inhibitor Ac-YVAD-cmk (40 mg kg ${ }^{-1}$ daily) starting treatment after 9 weeks of HFD (HFD+YVAD; $\left.n=15\right)$. Low-fat diet (LFD)-fed mice $(n=15)$ were included as a reference. (a) Representative photomicrographs of hematoxylin and eosin-stained liver sections. (b) Macrovesicular steatosis did not differ between groups. (c) HFD feeding induced pronounced microvesicular steatosis, which was reduced in HFD+YVAD. (d) HFD-induced hepatic triglycerides tended to be reduced in HFD+YVAD. All data are from the $t=21$ weeks time point and are mean \pm s.e.m. ${ }^{* * P}<0.01$ compared with HFD.

hepatic collagen deposition $(1.80 \pm 0.36$ in HFD, $0.68 \pm 0.17$ in HFDYVAD, $62 \%$ reduction, $P<0.01$, Figure $5 c$ ). Consistently, we observed a marked reduction ( $-49 \%)$ in expression of the stellate cell activation marker Acta2 alpha smooth muscle actin (aSMA); $1.65 \pm 0.23$ in HFD, $0.84 \pm 0.09$ in HFD-YVAD, $P<0.01$, Figure $5 \mathrm{~d}$ ). Collectively, these data indicate that therapeutic intervention with Ac-YVAD-cmk attenuates hepatocellular inflammation and activation of the primary collagen-producing cell type in the liver.

\section{DISCUSSION}

A growing body of evidence supports an important role for NLRP3 inflammasome activation in the development of obesity-related diseases such as insulin resistance/type 2 diabetes and NASH. However, the potential therapeutic value of inhibitors of the inflammasome/caspase- 1 to treat NASH remains unclear. Here we show that intervention with the caspase- 1 inhibitor Ac-YVAD-cmk reduces development of NASH and associated fibrosis. Importantly, efficacy of Ac-YVAD-cmk was demonstrated in mice with established obesity-associated hypertriglyceridemia, hypercholesterolemia, hyperglycemia and hyperinsulinemia.

We found that treatment with Ac-YVAD-cmk substantially retarded the progression of NAFLD, with reductions in hepatic steatosis, inflammation and fibrosis. In accordance with our observations, others have reported that genetic deletion of caspase-1 reduces HFD-induced hepatic triglyceride levels and lipogenic gene expression (Pparg, Srebp1c, Acc, Scd1) $)^{14}$ and deletion of NLRP3 attenuates HFD-induced hepatic steatosis with increased expression of fatty acid oxidation regulators. ${ }^{28}$ Furthermore, deletion of caspase- 1 was shown to reduce intestinal triglyceride absorption after an oral lipid load, with consequent reductions in the uptake and incorporation of these intestinally derived triglycerides into peripheral tissues. ${ }^{29}$ Together, these effects on hepatic lipid metabolism and intestinal lipid uptake may promote a reduction in hepatic steatosis in the absence of active caspase-1, as observed herein.
Although the effects of Ac-YVAD-cmk treatment on hepatic steatosis in the current study were modest, we did observe a strong effect on hepatic inflammation. In line with this, Dixon et al. have shown that deletion of caspase-1 leads to dissociation between hepatic triglyceride levels and inflammatory activity. ${ }^{13}$ Results from our analysis of hepatic inflammation indicate that the anti-inflammatory effects of Ac-YVAD-cmk treatment are attributable to an effect on the influx of MPO-positive neutrophilic cells, without affecting the expression of the monocyte/macrophage marker F4/80. Consistent with this, others report that caspase-1 deficiency does not affect HFD-induced hepatic F4/80 expression and suggest that caspase-1 may be of importance in regulating the sensitivity of Kupffer cells to activation rather than recruitment and/or proliferation of Kupffer cells in the liver. ${ }^{14}$ Furthermore, constitutively expressed hyperactive NLRP3 resulted in severe liver inflammation with many inflammatory foci composed predominantly of neutrophils, in the absence of an effect on F4/80 expression. ${ }^{15}$ Together, these results indicate that modulation of caspase- 1 expression or activity primarily influences the influx of neutrophils into the liver, which is considered a defining characteristic of human NASH. ${ }^{30}$ As neutrophils have the ability to release a potent cocktail of reactive oxygen species and proteases, they are a potential cause of extensive tissue damage that may contribute to amplification of the inflammatory response and development of hepatic fibrosis. ${ }^{31,32}$ More specifically, a recent study has shown that neutrophilic MPO promotes progression of NASH to fibrosis, potentiating oxidative stress, causing hepatocyte injury and activating hepatic stellate cells. ${ }^{33}$

In line with the observed reductions in hepatic inflammation and, more specifically, neutrophil infiltration, we observed a reduction in the development of hepatic fibrosis in Ac-YVADcmk-treated mice. Multiple lines of evidence indicate that caspase1 activation is required and essential for hepatic fibrogenesis. Watanabe et al. demonstrated that NLRP3 inflammasome activation in hepatic stellate cells results in the activation and production of collagen by these cells. ${ }^{34}$ Furthermore, they showed 

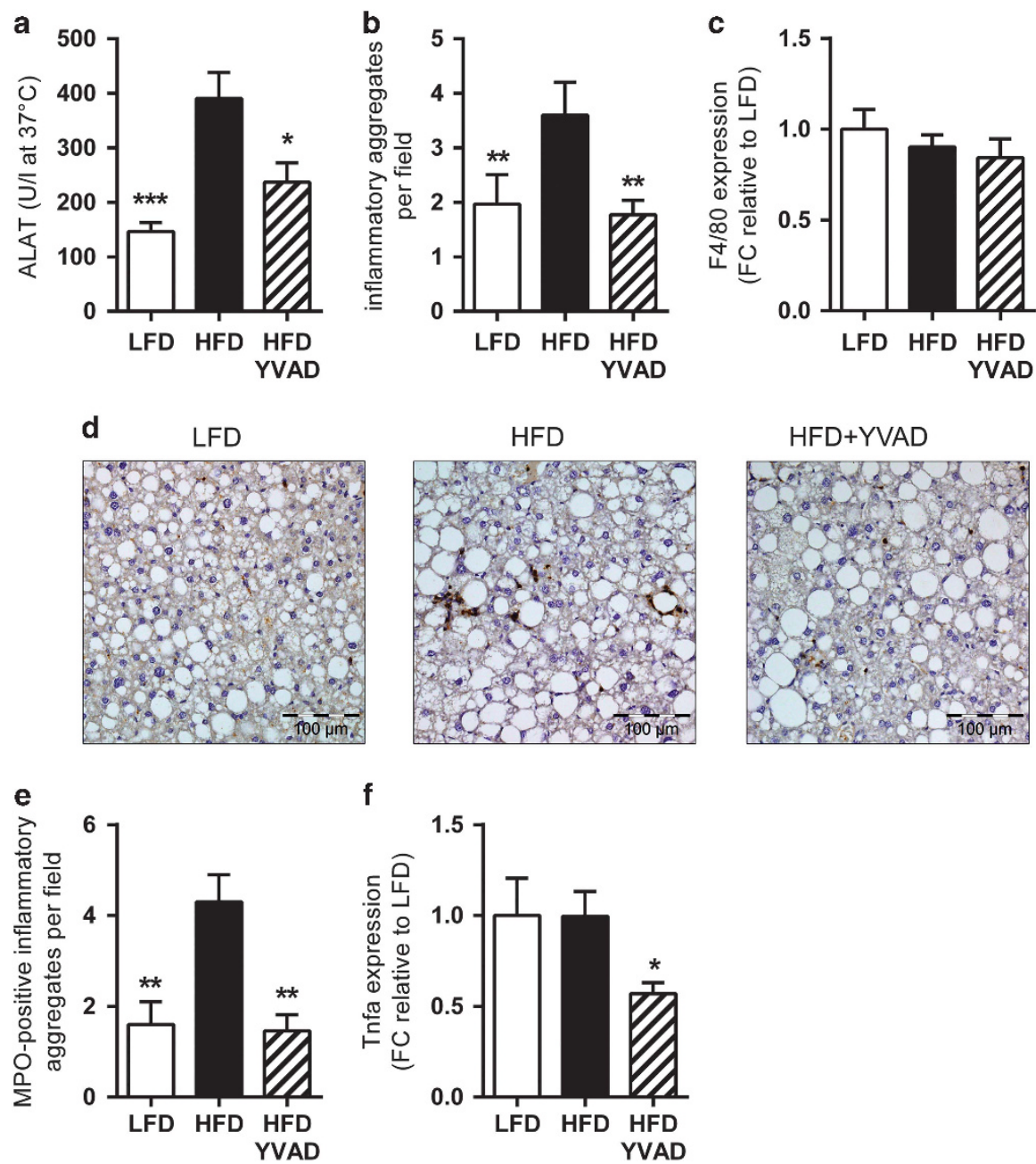

Figure 4. Effects of caspase-1 inhibition on hepatic inflammation. LDLR-/-.Leiden mice were fed a high-fat diet for 21 weeks (HFD; $n=15)$ or HFD+caspase-1 inhibitor Ac-YVAD-cmk (40 mg kg ${ }^{-1}$ daily) starting treatment after 9 weeks of HFD (HFD+YVAD; $\left.n=15\right)$. Low-fat diet (LFD)-fed mice $(n=15)$ were included as a reference. (a) HFD-induced plasma alanine aminotransferase (ALAT) was reduced in HFD+YVAD. (b) Lobular inflammation (number of inflammatory foci per x100 field) was induced by HFD, this induction was prevented by Ac-YVAD-cmk treatment. (c) F4/80 mRNA expression did not differ between groups. (d) Representative photomicrographs of immunohistochemical analysis of MPOpositive neutrophils. (e) Quantification of the number of MPO-positive inflammatory foci (per x100 field) revealed a clear induction in HFD, which was not apparent in YVAD. (f) Hepatic Tnf- $\alpha$ mRNA was reduced in HFD+YVAD. All data are from the $t=21$ weeks time point and are mean \pm s.e.m. ${ }^{*} P<0.05,{ }^{* *} P<0.01,{ }^{* * *} P<0.001$ compared with HFD.

that chemically induced (with $\mathrm{CCl}_{4}$ or Thioacetamide) hepatic stellate cell activation and liver fibrosis was reduced in mice deficient in NLRP3 or the inflammasome adaptor protein ASC. In line with this, Dixon et al. have shown reduced hepatic stellate cell activation and collagen deposition in Casp1-/ - mice fed an MCD diet $^{13}$ or a HFD. ${ }^{14}$ Similarly, hepatic stellate cell activation and collagen deposition were also reduced in NLRP3 - / - mice on a choline-deficient amino acid-defined diet, ${ }^{12}$ whereas mice that express constitutively hyperactive NLRP3 showed increased collagen deposition relative to wild-type mice. ${ }^{15}$ Altogether, these studies indicate that NLRP3 inflammasome activation is a generic process that is observed during fibrogenesis across a range of different (both chemically and dietary-induced) models of hepatic fibrosis. Our observations of reduced hepatic stellate cell activation and collagen deposition further corroborate these observations, and show that therapeutic intervention with a caspase- 1 inhibitor in the ongoing disease process can reduce development of hepatic fibrogenesis.

As obesity-associated insulin resistance is thought to play a causal role in the pathogenesis of $\mathrm{NASH}^{35}$ we studied the effects of caspase-1 inhibition in the LDLR-/-.Leiden model, which develops NAFLD in the context of insulin resistance. We found that treatment with Ac-YVAD-cmk retarded HFD-induced increases in fasting plasma glucose and insulin levels. This is in line with findings by others, who have shown improved insulin sensitivity in Casp1-/- mice. ${ }^{36,37}$ It is thought that this effect of caspase- 1 on insulin sensitivity may be mediated for a large part by the role that caspase- 1 plays in expanding WAT. Stienstra et al. $^{36}$ demonstrated that obesity-induced inflammation originating from expanding WAT is represented by inflammasome and caspase- 1 activation, which governs adipocyte differentiation and insulin sensitivity and contributes to insulin resistance. In vitro studies in mature human Simpson-Golabi-Behmel syndrome adipocytes showed that treatment with the caspase-1 inhibitor pralnacasan directly enhanced insulin signaling in these cells, indicating that caspase- 1 activation within adipocytes may have direct detrimental effects on insulin sensitivity of these cells. These effects were further substantiated in vivo in a proof-of-concept experiment, in which ob/ob mice were treated with pralnacasan for 2 weeks, ${ }^{36}$ showing that caspase- 1 inhibition can improve insulin sensitivity in vivo. 
a

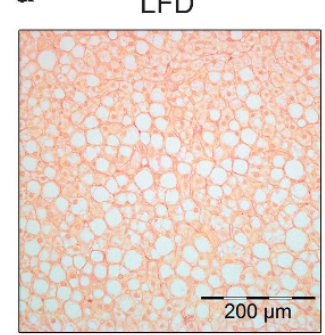

HFD

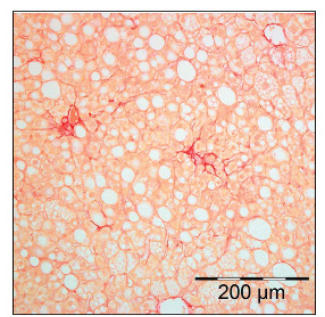

HFD+YVAD

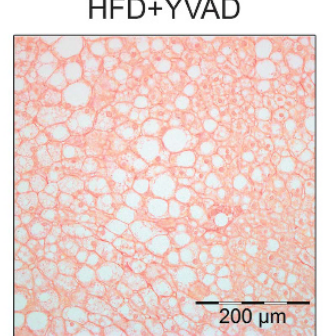

d

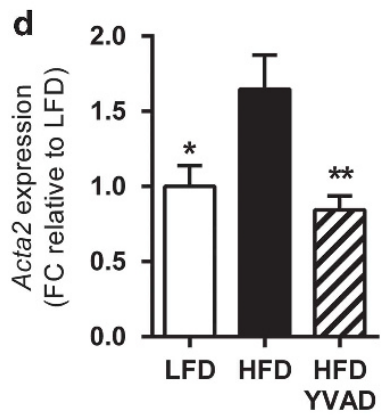

YNAD b

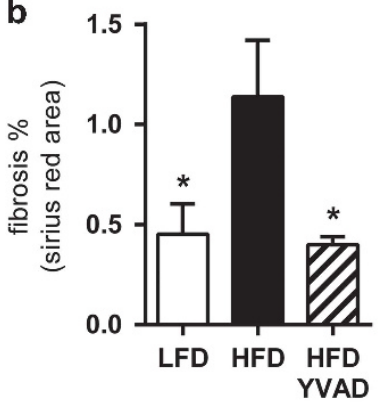

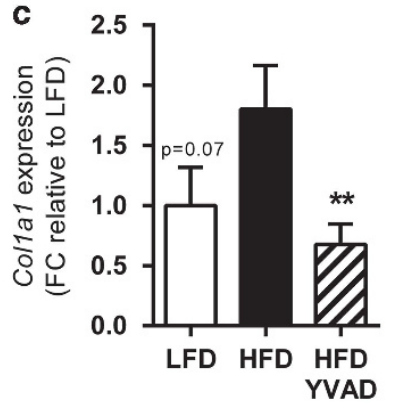

Figure 5. Effects of caspase-1 inhibition on hepatic fibrosis. LDLR-/-.Leiden mice were fed a high-fat diet for 21 weeks (HFD; $n=15$ ) or HFD +caspase-1 inhibitor Ac-YVAD-cmk (40 mg kg ${ }^{-1}$ daily) starting treatment after 9 weeks of HFD (HFD+YVAD; $\left.n=15\right)$. Low-fat diet (LFD)-fed mice $(n=15)$ were included as a reference. (a) Representative photomicrographs of Picro-Sirius red-stained liver sections show hepatopericellular fibrosis in HFD animals, which is reduced in HFD+YVAD. (b) Quantification of positively stained area in Picro-Sirius redstained liver sections show a clear induction of fibrosis in HFD, which is reduced in HFD+YVAD. (c) Col1a1 mRNA expression is reduced in HFD +YVAD. (d) mRNA expression of hepatic stellate cell activation marker Acta2 ( $\alpha-S M A)$ tended to be induced in HFD and was reduced in HFD + YVAD. All data are from the $t=21$ weeks time point and are mean \pm s.e.m. ${ }^{*} P<0.05,{ }^{* *} P<0.01$ compared with HFD.

Remarkably, we observed no elevation in plasma insulin in Ac-YVAD-cmk-treated mice during the ipGTT. There are several potential explanations for this phenomenon. First, we cannot exclude that insulin levels may have peaked between two of the time points of blood sampling, although this seems unlikely given the plasma half-life of insulin of around 10 min in mice. ${ }^{38}$ Second, it is possible that Ac-YVAD-cmk affected non-insulin-dependent glucose uptake. All cell types have the capacity to take up glucose independently of insulin, ensuring basal as well as hyperglycemiapromoted supply of glucose. As much as $75 \%$ of whole-body glucose uptake is considered to be operated through pathways that are non-insulin dependent, ${ }^{39}$ for instance through effects on cell membrane fluidity, which is known to influence glucose transporter activity. ${ }^{40}$ Third, Ac-YVAD-cmk may stimulate proteins and their actions downstream of the insulin receptor (at the postreceptor level or in the lower part of the insulin cascade) and may thus generate a more efficacious effect with the available insulin. ${ }^{39}$ The relatively high absolute insulin levels observed in Ac-YVADcmk-treated mice $\left(\sim 11 \mathrm{ng} \mathrm{ml}^{-1}\right)$ are in support of this notion. Another possible rationale may be found in an effect of Ac-YVAD$\mathrm{cmk}$ on the potency of the insulin molecules themselves (either through direct interaction with insulin or indirectly through its effects on caspase-1), in a manner similar to that which has been observed for instance for the chemokine MIF, which affects insulin conformation and thereby increases the potency of insulin molecules. ${ }^{41}$ Which of these mechanisms-alone or in concert-is responsible for the observed effects merits further investigation.

In the present study, reduced progression of insulin resistance and NAFLD development coincided with improvement of WAT inflammation in Ac-YVAD-cmk-treated animals. This corresponds with findings by others, who have shown that the absence of caspase- 1 reduces macrophage infiltration into WAT and protects against HFD-induced WAT inflammation. ${ }^{37}$ Inflammation of WAT (that is, macrophage infiltration and CLS formation) is increasingly considered to be a crucial event in the development of obesity- associated insulin resistance and NAFLD. ${ }^{42}$ Besides associative studies demonstrating associations between progressive NAFLD and WAT inflammation in obese subjects, ${ }^{43,44}$ experimental studies have shown that obesity-associated inflammation in WAT precedes hepatic inflammation in HFD-fed mice. ${ }^{45,46}$ Surgical removal of inflamed WAT provided evidence for a causal role of inflamed WAT in the progression of $\mathrm{NASH}^{47}$ The observed reduction of WAT inflammation herein may thus indirectly have contributed to the attenuation of NASH development by Ac-YVAD-cmk.

Overall, we show that intervention with the caspase-1 inhibitor Ac-YVAD-cmk improves insulin resistance and retards the progression of NASH and fibrosis development in male LDLR-/-. Leiden mice. Data from this study further support the importance of inflammasome/caspase-1 in the development of insulin resistance and NASH and demonstrate that therapeutic intervention in the already ongoing disease process is feasible.

Supplementary information is available at International Journal of Obesity's website.

\section{CONFLICT OF INTEREST}

The authors declare no conflict of interest.

\section{ACKNOWLEDGEMENTS}

We thank Simone van der Drift-Droog, Erik Offerman and Karin Toet for their excellent technical assistance. This work was funded by the TNO research program 'Enabling Technology Systems Biology'. MCM received funding from TI Food and Nutrition, a public-private partnership on pre-competitive research in food and nutrition.

\section{REFERENCES}

1 Fabbrini E, Sullivan S, Klein S. Obesity and nonalcoholic fatty liver disease: biochemical, metabolic, and clinical implications. Hepatology 2010; 51: 679-689. 
2 Birkenfeld AL, Shulman GI. Nonalcoholic fatty liver disease, hepatic insulin resistance, and type 2 diabetes. Hepatology 2014; 59: 713-723.

3 Tiniakos DG, Vos MB, Brunt EM. Nonalcoholic fatty liver disease: pathology and pathogenesis. Annu Rev Pathol 2010; 5: 145-171.

4 Angulo P. Nonalcoholic fatty liver disease. N Engl J Med 2002; 346: 1221-1231.

5 Charlton MR, Burns JM, Pedersen RA, Watt KD, Heimbach JK, Dierkhising RA. Frequency and outcomes of liver transplantation for nonalcoholic steatohepatitis in the United States. Gastroenterology 2011; 141: 1249-1253.

6 Hotamisligil GS. Inflammation and metabolic disorders. Nature 2006; 444 860-867.

7 Exley MA, Hand L, O'Shea D, Lynch L. Interplay between the immune system and adipose tissue in obesity. $J$ Endocrinol 2014; 223: R41-R48.

8 Horng T, Hotamisligil GS. Linking the inflammasome to obesity-related disease. Nat Med 2011; 17: 164-165.

9 Csak T, Ganz M, Pespisa J, Kodys K, Dolganiuc A, Szabo G. Fatty acid and endotoxin activate inflammasomes in mouse hepatocytes that release danger signals to stimulate immune cells. Hepatology 2011; 54: 133-144.

10 Wen H, Gris D, Lei Y, Jha S, Zhang L, Huang MT et al. Fatty acid-induced NLRP3ASC inflammasome activation interferes with insulin signaling. Nat Immunol 2011; 12: $408-415$.

11 Gross O, Thomas CJ, Guarda G, Tschopp J. The inflammasome: an integrated view. Immunol Rev 2011; 243: 136-151.

12 Wree A, McGeough MD, Pena CA, Schlattjan M, Li H, Inzaugarat ME et al. NLRP3 inflammasome activation is required for fibrosis development in NAFLD. J Mol Med 2014; 92: 1069-1082.

13 Dixon LJ, Berk M, Thapaliya S, Papouchado BG, Feldstein AE. Caspase-1-mediated regulation of fibrogenesis in diet-induced steatohepatitis. Lab Invest 2012; 92: 713-723.

14 Dixon LJ, Flask CA, Papouchado BG, Feldstein AE, Nagy LE. Caspase-1 as a central regulator of high fat diet-induced non-alcoholic steatohepatitis. PLoS One 2013; 8 : e56100.

15 Wree A, Eguchi A, McGeough MD, Pena CA, Johnson CD, Canbay A et al. NLRP3 inflammasome activation results in hepatocyte pyroptosis, liver inflammation, and fibrosis in mice. Hepatology 2014; 59: 898-910.

16 Liang W, Menke AL, Driessen A, Koek GH, Lindeman JH, Stoop R et al. Establishment of a general NAFLD scoring system for rodent models and comparison to human liver pathology. PLoS One 2014; 9: e115922.

17 Radonjic M, Wielinga PY, Wopereis S, Kelder T, Goelela VS, Verschuren L et al. Differential effects of drug interventions and dietary lifestyle in developing type 2 diabetes and complications: a systems biology analysis in LDLr-/- mice. PLoS One 2013; 8: e56122.

18 Mulder P, Liang W, Wielinga PY, Verschuren L, Toet K, Havekes LM et al. Macrovesicular steatosis is associated with development of lobular inflammation and fibrosis in dietinduced non-alcoholic steatohepatitis (NASH). Inflamm Cell Signal 2015; 2: e804.

19 Lipinska K, Malone KE, Moerland M, Kluft C. Applying caspase-1 inhibitors for inflammasome assays in human whole blood. J Immunol Methods 2014; 411: 66-69.

20 Osuka A, Hanschen M, Stoecklein V, Lederer JA. A protective role for inflammasome activation following injury. Shock 2012; 37: 47-55.

21 Suzuki H, Sozen T, Hasegawa Y, Chen W, Zhang JH. Caspase-1 inhibitor prevents neurogenic pulmonary edema after subarachnoid hemorrhage in mice. Stroke 2009; 40: 3872-3875.

22 Liang W, Lindeman $\mathrm{JH}$, Menke AL, Koonen DP, Morrison M, Havekes LM et al. Metabolically induced liver inflammation leads to NASH and differs from LPS- or IL-1beta-induced chronic inflammation. Lab Invest 2014; 94: 491-502.

23 Bligh EG, Dyer WJ. A rapid method of total lipid extraction and purification. Can J Biochem Physiol 1959; 37: 911-917.

24 Lowry OH, Rosebrough NJ, Farr AL, Randall RJ. Protein measurement with the Folin phenol reagent. J Biol Chem 1951; 193: 265-275.

25 Morrison MC, Liang W, Mulder P, Verschuren L, Pieterman E, Toet K et al. Mirtoselect, an anthocyanin-rich bilberry extract, attenuates non-alcoholic steatohepatitis and associated fibrosis in ApoE *3Leiden mice. J Hepatol 2015; 62: 1180-1186.

26 Hubscher SG. Histological assessment of non-alcoholic fatty liver disease. Histopathology 2006; 49: 450-465.

27 Rensen SS, Slaats Y, Nijhuis J, Jans A, Bieghs V, Driessen A et al. Increased hepatic myeloperoxidase activity in obese subjects with nonalcoholic steatohepatitis. Am J Pathol 2009; 175: 1473-1482.

28 Vandanmagsar B, Youm YH, Ravussin A, Galgani JE, Stadler K, Mynatt RL et al. The NLRP3 inflammasome instigates obesity-induced inflammation and insulin resistance. Nat Med 2011; 17: 179-188.
29 van Diepen JA, Stienstra R, Vroegrijk IO, van den Berg SA, Salvatori D, Hooiveld GJ et al. Caspase-1 deficiency in mice reduces intestinal triglyceride absorption and hepatic triglyceride secretion. J Lipid Res 2013; 54: 448-456.

30 Brunt EM, Janney CG, Di Bisceglie AM, Neuschwander-Tetri BA, Bacon BR Nonalcoholic steatohepatitis: a proposal for grading and staging the histological lesions. Am J Gastroenterol 1999; 94: 2467-2474.

31 Kubes P, Mehal WZ. Sterile inflammation in the liver. Gastroenterology 2012; 143: 1158-1172.

32 Xu R, Huang H, Zhang Z, Wang FS. The role of neutrophils in the development of liver diseases. Cell Mol Immunol 2014; 11: 224-231.

33 Pulli B, Ali M, Iwamoto Y, Zeller MW, Schob S, Linnoila JJ et al. Myeloperoxidasehepatocyte-stellate cell cross talk promotes hepatocyte injury and fibrosis in experimental nonalcoholic steatohepatitis. Antioxid Redox Signal 2015; 23 1255-1269.

34 Watanabe A, Sohail MA, Gomes DA, Hashmi A, Nagata J, Sutterwala FS et al Inflammasome-mediated regulation of hepatic stellate cells. Am J Physiol 2009; 296: G1248-G1257.

35 Pagano G, Pacini G, Musso G, Gambino R, Mecca F, Depetris N et al. Nonalcoholic steatohepatitis, insulin resistance, and metabolic syndrome: further evidence for an etiologic association. Hepatology 2002; 35: 367-372.

36 Stienstra R, Joosten LA, Koenen T, van Tits B, van Diepen JA, van den Berg SA et al. The inflammasome-mediated caspase- 1 activation controls adipocyte differentiation and insulin sensitivity. Cell Metab 2010; 12: 593-605.

37 Stienstra R, van Diepen JA, Tack CJ, Zaki MH, van de Veerdonk FL, Perera D et al. Inflammasome is a central player in the induction of obesity and insulin resistance. Proc Natl Acad Sci USA 2011; 108: 15324-15329.

38 Cresto J, Lavine R, Buchly M, Penhos J, Bhathena S, Recant L. Half life of injected 125I-insulin in control and ob/ob mice. Acta Physiol Latino Am 1976; 27: 7-15.

39 Wiernsperger NF. Is non-insulin dependent glucose uptake a therapeutic alternative? Part 1: physiology, mechanisms and role of non insulindependent glucose uptake in type 2 diabetes. Diabetes Metab 2005; 31: 415-426.

40 Pilch PF, Thompson PA, Czech MP. Coordinate modulation of D-glucose transport activity and bilayer fluidity in plasma membranes derived from control and insulin-treated adipocytes. Proc Natl Acad Sci U S A 1980; 77: 915-918.

41 Vujicic M, Senerovic L, Nikolic I, Saksida T, Stosic-Grujicic S, Stojanovic I. The critical role of macrophage migration inhibitory factor in insulin activity. Cytokine 2014; 69: 39-46.

42 Esser N, Legrand-Poels S, Piette J, Scheen AJ, Paquot N. Inflammation as a link between obesity, metabolic syndrome and type 2 diabetes. Diabetes Res Clin Pract 2014; 105: 141-150.

43 Cancello R, Tordjman J, Poitou C, Guilhem G, Bouillot JL, Hugol D et al. Increased infiltration of macrophages in omental adipose tissue is associated with marked hepatic lesions in morbid human obesity. Diabetes 2006; 55: 1554-1561.

44 Tordjman J, Poitou C, Hugol D, Bouillot J-L, Basdevant A, Bedossa P et al. Association between omental adipose tissue macrophages and liver histopathology in morbid obesity: Influence of glycemic status. J Hepatol 2009; 51: 354-362.

45 Liang W, Tonini G, Mulder P, Kelder T, van Erk M, van den Hoek AM et al. Coordinated and interactive expression of genes of lipid metabolism and inflammation in adipose tissue and liver during metabolic overload. PLoS One 2013; 8: e75290.

46 van der Heijden RA, Sheedfar F, Morrison MC, Hommelberg PP, Kor D, Kloosterhuis NJ et al. High-fat diet induced obesity primes inflammation in adipose tissue prior to liver in C57BL/6j mice. Aging 2015; 7: 256.

47 Mulder P, Morrison MC, Wielinga PY, van Duyvenvoorde W, Kooistra T, Kleemann R. Surgical removal of inflamed epididymal white adipose tissue attenuates the development of non-alcoholic steatohepatitis in obesity. Int J Obes 2015; 40: 675-684.

This work is licensed under a Creative Commons AttributionNonCommercial-NoDerivs 4.0 International License. The images or other third party material in this article are included in the article's Creative Commons license, unless indicated otherwise in the credit line; if the material is not included under the Creative Commons license, users will need to obtain permission from the license holder to reproduce the material. To view a copy of this license, visit http:// creativecommons.org/licenses/by-nc-nd/4.0/

Supplementary Information accompanies this paper on International Journal of Obesity website (http://www.nature.com/ijo) 